\section{The ART AND SCIENCE OF RECORDING THE ENVIRONMENT}

Leah Barclay, Griffith University, Brisbane, Australia. Email: <1.barclay@griffith.edu.au>.

Toby Gifford, Griffith University, Brisbane, Australia. Email: <t.gifford@griffith.edu.au>.

See $<w w w . m i t p r e s s j o u r n a l s . o r g / t o c / l e o n / 51 / 2>$ for supplemental files associated with this issue.

Submitted: 21 October 2016

\section{Abstract}

The recent emergence of the interdisciplinary fields of ecoacoustics and sound studies has resulted in a dramatic increase in both artists and scientists engaged in the practice of audio field recording for a diversity of purposes. The recording techniques used vary substantially reflecting differing loci of interest. We argue that both fields could benefit from greater cross-fertilization, and enhanced discussion of existing field recording practices. We suggest acoustic ecology as a field provides a natural home for such interdisciplinary exchanges, and discuss our application of Acoustic Ecology in the Biosphere Soundscapes project.

Over the past five years, two interdisciplinary fields of environmental acoustics have crystallised on either side of the arts / science divide - in the sciences ecoacoustics, and in the humanities sound studies. Neither field is new per se, with examples of such work historically to be found emerging from existing disciplines such as bioacoustics, acoustic ecology, landscape ecology and cultural theory. However, their relatively recent solidification as distinct fields has resulted in a substantial increase in both artists and scientists engaged in the practice of acoustic field recording.

Approaches to field recording vary dramatically between and even within these fields, and sometimes are developed nescient of existing bodies of practice in nature recording. Conversely, practical requirements of these new fields are often at odds with purely audiophile considerations. Consequently there appears to be a lack of productive exchange of ideas between past and present, and across related disciplines.

\section{Ecoacoustics}

Ecoacoustics [1] draws on a number of existing sonic disciplines - including bioacoustics, acoustic ecology and soundscape ecology — whose boundaries are sometimes contested. A point of contention, and in some cases demarcation, is the role of aesthetics and human perception, with epistemological tensions mirroring C.P. Snow's observation of the "Two Cultures" of arts and science.

As a practical example of disciplinary divergence within this constellation, consider differing approaches to sound quality. In bioacoustics it is not uncommon to take field recordings in 'mono' at low sampling rates, for reasons both practical-cost, data storage, power consumption, bandwidth; and theoreticalmost acoustic indices and classifier algorithms are monophonic, and operate in the frequency domain, so that the high sampling rates needed to encode complex spatial or reverberant information are redundant. In contrast, for acoustic ecologists stereo recording is the de facto standard, high sampling rates preferred, and more complex techniques such as binaural or ambisonic recording common.

At root this divergence relates to the role of human perception in the process: typically bioacoustics seeks to remove subjective perception whilst acoustic ecology privileges it. Yet these approaches need not be mutually exclusive. Computational Auditory Scene Analysis remains in infancy - the best machine listening systems still are no match for the human auditory perception of complex soundscapes. The human mind possesses remarkable pattern perception mechanisms which may assist the process of exploration and discovery, even if a purely objective theory validation process is also sought.

\section{Sound Studies}

The field of sound studies [2] has historical roots primarily in media and communication theory, with a cultural theory perspective. In confluence with an increasing interest in sound from the urban planning and architectural science fields, sound studies looks beyond the traditional categorization of 'sound as noise' in urban planning, to a more nuanced study of sound and place recognizing its potentially positive aspects, cultural determinedness and social importance. Sound studies theorists may not necessarily have experience in field recording, or else may hail from a media production background in which a studio-based metaphor of control over the sonic environment prevails. Natural environment recording entails a different perspective, the embrace of which may aid theorists to develop new insights.

\section{Interdisciplinary Approaches}

The structure of UNESCO biosphere reserves, simultaneously involving ecological, social and cultural considerations, lends itself to interdisciplinary studies that cross science, arts and humanities. This is evident through Biosphere Soundscapes, an initiative established in 2011 to map the changing soundscapes of UNESCO biosphere reserves and explore the creative possibilities of acoustic ecology. This project is designed to inspire communities to listen to the environment and explore the value of sound as a measure for environmental health and ecological engagement. It is delivered through immersive residencies with artists and scientists, intensive masterclasses and a diversity of creative projects spanning four continents.

The Biosphere Soundscapes residencies involves 10-days of immersive field recording with a selected group of participants. The project adopts diverse field recording approaches, ranging from long durational remote recordings to in-situ sessions involving high-quality equipment and techniques. The resulting recordings are used for scientific enquiry and biodiversity analysis and become source material for diverse artistic purposes, ranging from installations and compositions, to educational material and radio productions. The residencies involve critical enquiry and theoretical discussions, providing artists and scientists with a platform to share ideas and experiences. This process consistently reveals the value of interdisciplinary collaborations and has resulted in partnerships and research underpinned by aesthetic and technical approaches.

Biosphere Soundscapes embraces the possibilities of these emerging fields and calls for greater cross-fertilization, and enhanced discussion of existing field recording practices. We suggest the established interdisciplinary field of acoustic ecology and its associated global networks as a viable lens for ongoing exchange and exploration that encompasses the social, cultural and ecological contexts of our sonic environment.

\section{References and Notes}

1. Jérôme Sueur and Almo Farina, "Ecoacoustics: the Ecological Investigation and Interpretation of Environmental Sound," Biosemiotics, 8 (2015) pp. 493-502.

2. Music, Sound and Space: Transformations of Public and Private Experience, Georgina Born, ed. (Cambridge: Cambridge Univ. Press, 2015). 